ORIGINAL ARTICLE

\title{
Best practice for the management of older people with dementia in the acute care setting: a review of the literature
}

\author{
Wendy Moyle RN PhD \\ Professor of Nursing, Research Centre for Clinical and Community Practice Innovation, Griffith University, Nathan, Brisbane, \\ Australia
}

Rachel Olorenshaw RN BN

Research Assistant, Research Centre for Clinical and Community Practice Innovation, Griffith University, Nathan, Brisbane, Australia

\author{
Marianne Wallis RN PhD \\ Professor of Clinical Nursing Research, Research Centre for Clinical and Community Practice Innovation, Griffith University, \\ and Gold Coast Health Service District, Gold Coast, Australia
}

Sally Borbasi RN PhD

Professor of Nursing, Research Centre for Clinical and Community Practice Innovation, Griffith University, Logan, Brisbane, Australia

Submitted for publication: XX Xxxxx 200x

2Accepted for publication: XX Xxxxx 200x

Correspondence:
Wendy Moyle
Research Centre for Clinical and Community
Practice Innovation
Griffith University
Nathan
Brisbane
Australia
Telephone: +61 737355526
E-mail: w.moyle@griffith.edu.au

Correspondence:

MOYLE W., OLORENSHAW R., WALLIS M. \& BORBASI S. (2008) International Journal of Older People Nursing 3, 1-10

Best practice for the management of older people with dementia in the acute care setting: a review of the literature

Aim. This paper reviews the theoretical and research-based literature related to the management of people with chronic confusion as a consequence of dementia in the acute care setting.

Background. People aged 65 years and over are at increased risk of poor outcomes when admitted to the acute care setting as a result of comorbity and mismanagement of their chronic confusion. The challenge of caring for people with dementia in acute care is one that requires special attention.

Results. The theoretical literature outlines a number of principles of care necessary for best practice in the care of people with dementia. A number of different models of care are reported in the literature and some evaluative research has been undertaken to assess the benefits of the different models.

Conclusion. There are a number of interventions that if put into place in acute care may improve care of people with dementia and reduce the burden of care. There is emerging evidence that interventions such as staff education, skilled expertise, standardized care protocols and environmental modification help to meet the needs of people with dementia in acute hospital settings. This paper adds to our current understanding of management of dementia in the acute care setting, an area that demonstrates the need to move from descriptive to intervention studies to ensure evidence for care of persons with a dementing condition.

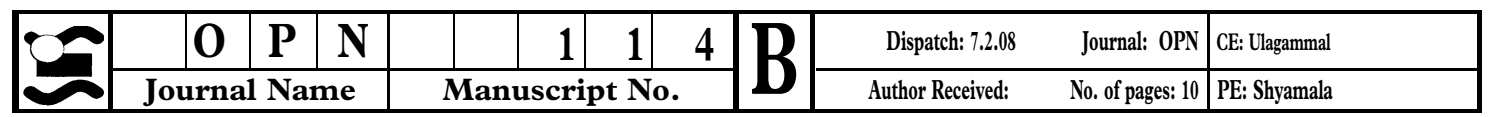


Key words: acute care, best practice, care models, chronic confusion, cognitive impairment, dementia

\section{Introduction}

Dementia is a syndrome characterized by specific signs and symptoms of a chronic nature, such as disturbed memory, thinking, orientation, personality and behavioural changes, comprehension, calculation, learning capacity, language and judgement, and which indicate progressive brain malfunction (Draper, 2004). There are a number of different dementias. Alzheimer's disease is the most common form of dementia, with a prevalence rate of around $10 \%$ of the population aged 65 years and older and approximately 50\% for those aged 85 years and older (Australian Institute of Health and Welfare (AIHW) 2004). Cognitive impairment and confusion as a result of dementia is a significant comorbidity among hospitalized older people. In Australia, for example, dementia was reported as a principal or additional diagnosis for $1.2 \%$ of discharged hospital patients (AIHW, 2004). Dementia is associated with an increase in use of medical services and longer hospital stay (Gutterman et al., 1999; AIHW, 2004), high risk of institutionalisation (AIHW, 2004) and disruptive behaviour, which is often cited as a significant problem that adds to the challenges of caregiving (Kolanow-

3ski et al., 2002; Chrzescijanski et al., 2007). Care of the older person with dementia in an acute hospital setting, presents care staff with a special challenge, especially the need to care for the individual's personhood as well as management of their disruptive behaviours such as wandering and vocalization. The management of such episodes impacts on outcomes for these patients and their relatives or carers and frequently influences the care of others within the hospital ward.

Unfortunately, many hospitals are not designed to care for people who are cognitively impaired. This leads not only to patient safety issues, but also contributes to the burden of care. In the hospital setting it is likely that the acute problem for which the person is admitted will become the main priority for care (Tolson et al., 1999). However, in order to achieve best practice standards of care for the person with dementia, there is a need for specific care for people with chronic confusion to be combined with acute care practices (Hart et al., 2002). Research based evidence will assist health professionals in making decisions about the care for people with chronic confusion, policy makers and organizations such as the Alzheimer's Society to produce practice guidelines and to identify knowledge gaps that require further research (Courtney et al., 2005). New guidelines were recently released in the UK to help care staff support those people living with dementia and their carers (Hunt, 2007). The guidelines provide support for the range of services that such people might need.

This literature review aims to examine the principles that underpin current best practice recommendations for the care of people with dementia in the acute setting and to assess the evidence for the efficacy of specific models of care designed to incorporate these principles. Although many of the principles described in this review are relevant to long term care, the acute setting has a number of facets that accentuate the challenges of care of people with chronic confusion and therefore need to be considered separately. These factors include an environment that is culturally ill prepared, not purpose built for or sufficiently aware of the essentials of care for people with dementia, and whose focus is the acute condition, an emphasis on technology and a majority of patients who are acutely ill. Secondly, this review has avoided where possible reference to literature that focuses only on acute confusion as the treatment and care of the person with delirium is considered to include care that falls outside of the essentials of care of the person with chronic confusion that is related to dementia.

\section{Methods}

A review of literature published in English between 1986 and 2006 is presented in this paper. Our search of the literature focused on peer reviewed papers reporting on best practice principles for the care of people with dementia in the acute care setting and on research evaluating interventions or models of care for older people with chronic confusion in acute care. Papers included in this review were chosen according to the following criteria:

\section{Inclusion criteria}

- main focus on care models and/or principles of care of dementia in older people in acute care.

- published in a peer reviewed journal between 1986 and 2006.

- paper describes the evidence basis for the model of care and/or principles of care of older people with cognitive impairment/chronic confusion in acute care. 


\section{Exclusion criteria}

- main focus was not on acute care.

- focus is on care of older people with acute confusion.

- published in a non-peer reviewed journal.

- evidence basis for principles or model of care not explicated.

\section{Search strategy}

Four major databases, Cumulative Index of Nursing and Allied Health Literature (CINAHL), Proquest, PsychLit and MEDLINE were searched using a variety of search terms including, 'dementia care', 'acute care', 'models of dementia care', 'chronic confusion', 'cognitive impairment'. A manual search of the reference lists in each identified paper helped to uncover further relevant papers. The search found 48 papers related to the search strategy. Each paper was checked against the inclusion criteria and this resulted in the 31 papers that are discussed in this paper. The following discussion of the findings commences with an identification of the common themes of care management followed closely by a critique of the selected models of care.

\section{Findings}

\section{Care of older people with dementia in the acute care setting}

Common themes gleaned from the literature on care of older people with cognitive impairment as a result of dementia in the acute care setting provide some consensus on patient management. They include; the importance of early detection and assessment; knowledge and attitudes of nursing staff; focused communication; a reduction in stressors; familiarity that includes family and carer involvement and a multidisciplinary approach. Such themes offer grounding for further research and the development of guidelines that address the unique needs of older people with dementia undergoing treatment for comorbidity in the acute care setting.

\section{Detection and assessment}

The incidence of dementia is on the increase as a result of an ageing population and as a consequence the prevalence of people with dementia in acute care will also be on the rise. Although the prevalence of people with dementia in acute care is not commonly known, it may be as many as one third of all older patients, although their dementia may not have been formally diagnosed (Mezey \& Maslow, 2004). Having a dementing condition may also put the person at increased risk of acute confusion, as the incidence of delirium is greater in people with dementia (Weber et al., 2004). The average incidence rate for acute confusion among hospitalized older people over the age of 65 is $40 \%$ (Weber et al., 2004). However, this may be a conservative figure as Fick and 4 Foreman (2000) suggest that as many as seven out of 10 patients with delirium are going undetected. Failure to detect dementia and delirium will affect the choice of treatment and strategies used to care for and manage these people.

Voyer et al. (2006) recommend that when trying to detect delirium in people with dementia it is important to monitor attention, thinking, orientation memory and the fluctuation of the symptoms rather than relying on psychomotor symptoms such as hypoactivity and hyperactivity. The documentation of symptoms of confusion is critical and according to Voyer et al. (2006) recording these symptoms as present or absent is inadequate and makes detection of delirium in older people with dementia difficult as fluctuations become impossible to notice. There are a variety of mental status/neurologic assessment tools available to assist nurses with assessment such as Folstein Mini Mental State Examination (MMSE) (Folstein et al., 1975). However, it is essential that nurses who use such tools are adequately trained to carry out the assessment (Lemiengre et al., 2006) and that they are aware of the limitations of the tool (Foreman \& Zane, 1996).

\section{Nurses' knowledge and attitudes}

Nolan (2006) reported on a qualitative study that explored nurses' experiences of caring for older people with dementia in an acute care setting. The sample of seven nurses found that the meaning of the caring experience was related to the personhood of the nurse and the patient. Although participants reported on the contextual challenges of the acute environment the author concluded that nurses lack specific knowledge about dementia and this challenged the operationalisation of the care process.

Disruptive behaviour by confused older people can impact on the time management and ward routines of nurses (Borbasi et al., 2006; Cunningham \& Archibald, 2006). It can also affect nurses' experience of caring when ethical decisions have to be made in stressful working environments such as the acute setting (Eriksson \& Saveman, 2002). Acute care nurses have reported using force, abuse and neglect to manage patient behaviour and some nurses have reported being afraid of people with dementia (Eriksson \& Saveman, 2002). Such management may occur when nurses lack the confidence and competence to care for patients' special needs (Pritchard \& Dewing, 2001). Furthermore, burnout of nursing staff caring for people who display disruptive 
behaviour is not uncommon (Eriksson \& Saveman, 2002). Nurses' knowledge about dementia (McCloskey, 2004) as well as their attitudes towards ageing (Pritchard \& Dewing, 2001; McCarthy, 2003a,b) can impact on nurses' experience and patient outcomes (Pfaff, 2002; Cunningham, 2006a).

According to the literature, nurses' knowledge could be improved in a number of areas. These include; a greater understanding of the clinical syndrome of dementia and how it differs from delirium (Fick \& Foreman, 2000; Poole, 2003); how to effectively assess mental status (Fick \& Foreman, 2000; Pritchard \& Dewing, 2001); factors that contribute to cognitive decline during acute illness and hospital admission (McCloskey, 2004); strategies to prevent cognitive decline (Cunningham, 2006a); and how to manage older people experiencing delirium and or dementia (Cunningham \& Archibald, 2006).

Nurses' attitude towards confused older people has been identified as a barrier to optimal care (Pritchard \& Dewing, 2001; McCarthy, 2003a,b). McCarthy (2003a) used a dimensional analysis approach to explore clinical reasoning of nurses caring for confused older people in the hospital setting. McCarthy (2003a) concluded that nurses' philosophical perspectives on ageing influence how they characterize, judge and deal with older adults. Furthermore she found that the predominant philosophical perspective amongst staff might predict how well they will perform when caring for large numbers of older adults, such as, in the hospital setting. She concluded three distinct perspectives were held by nurses; the decline perspective; vulnerable perspective; and healthful perspective (McCarthy, 2003a).

Nurses who hold the decline perspective undertake consequential reasoning, that is, if people are old they are confused and cognitive impairment is seen as inevitable among older people. The vulnerable perspective involves ambivalent or uncertain reasoning. Ageing is considered a time when the threat of disease and ill health is constant and although not inevitable both are likely to occur. The healthful perspective involves diagnostic reasoning. These nurses consider ageing to be a normal process and view older adults as essentially well. Cognitive decline is considered to be pathological and unusual. Nurses with a healthful perspective, in contrast to those in the other two groups, were able to pick up on early signs of confusion and take quick action and had an understanding of the differences between dementia and delirium. They also believed that in most cases of acute confusion an underlying pathophysiological cause was likely, however, they considered chronic confusion as possible when other possible causes for the confusion were ruled out (McCarthy, 2003a). Strategies such as education, organizational, administrative, and peer support, which aim to improve the thinking and behaviour of nurses, were recommended (McCarthy, 2003a).

\section{Communication}

Dementia impairs the individuals' ability to understand and express information, plan and solve problems (Draper, 2004). Other dementia-related impairments such as memory loss, as well as the hospital setting and acute illness tend to exacerbate communication problems (Frazier-Rios \& Zembrzuski, 2005). Disruptive behaviour is often a form of communication for the person with dementia (Kolanowski et al., 2002). Unmet needs of the older person may be expressed through behaviours such as agitation, restlessness and aggression as well as vocalizations that are repetitive and show changes in tone and urgency (Kolanowski et al., 2002; Frazier-Rios \& Zembrzuski, 2005). Many practitioners have used validation therapy as developed by Naomi Feil (1972) for older people with dementia to assist with communication. The therapy accepts the truth of another's experience and uses behavioural and psychotherapeutic methods to meet the needs of people with dementia. This therapy has attracted a lot of followers as well as criticism and a separate review of this therapy found very little research available. A Cochrane systematic review (Neal \& Barton Wright, 2003) identified three papers that meet the inclusion criteria (Peoples, 1982; Robb, 1986; Roseland, 1997). Two significant results were found; Peoples (1982) explored validation therapy vs. usual care and found that participant's behaviour at 6 weeks favoured validation therapy (MD 5.97, 95\% CI, $P=0.0007$ ). 8 Toseland (1997) explored validation therapy vs. social contact and identified that depression at 12 months favoured validation (MS 4.01, 95\% CI, $P=0.04$ ). Despite these results, there were no statistically significant differences between the items and the authors concluded that there was insufficient evidence to draw any conclusion about efficacy of validation therapy. Cunningham (2006a) suggests that behaviour should be assessed retrospectively by describing what the nurses observe using the antecedents, behaviour and consequences (ABC) analysis of behaviour tool. Such a method may help to stop practitioners inappropriately labelling and blaming people with dementia, and to explore how they might adapt their practice and the environment rather than to change the person. A number of authors (e.g. Pritchard \& Dewing, 2001; McCloskey, 2004; Frazier-Rios \& Zembrzuski, 2005; Cunningham, 2006a) offer strategies that aim to facilitate communication (see Table 1).

\section{External stressors}

The hospital environment produces multiple and competing stimuli, which can be unsettling for people who have 
Table 1 Strategies to facilitate communication as described in the literature

Approach patient calmly, gently and relaxed

Speak directly to the patient even if unable to respond

Remain calm if patient becomes agitated

Quieten area of remove excess stimuli during communication

Allow ample response time before repeating information

Limit choices

Observe and value both verbal and non-verbal communication
Use person's preferred name

Avoid moving or walking around during conversation

Repeat sentences using the same words

Use short, simple sentences and a soft tone

Ask simple questions that require a short answer, e.g. yes/no

Model the desired behaviour impaired cognition. Unfortunately, many hospitals are not designed to care for older people who are cognitively impaired or confused as a result of dementia. In the past, hospitals restrained people who were confused to prevent wandering and apparent injury. However, numerous studies have found that restraining people may impose an even greater risk and may worsen confusion (Price et al., 2005; Borbasi et al., 2006). Experts believe that hospitals must adapt to the needs of an ageing population (Jimenez-Vilchez et al., 2004). Odours, noise and décor contribute to the information processing of people with dementia and therefore should be considered for modification (Hanley, 2004). Environmental interventions that can be implemented in the hospital setting include: provision of memory cues, that is, clocks, calendars and photos; reducing clutter, that is, remove unnecessary equipment; avoid unnecessary environmental changes; keep glasses and hearing aids in reach; provide purposeful activity. More structural methods include: adequate and appropriate lighting; lowering signs; considering choice of colours (red, orange and yellow are more easily seen by older people) (Hanley, 2004; Cunningham, 2006a). The ideal environment for people who are confused can be difficult to achieve in the hospital setting, however, an effort should be made to maintain a calm, well organized, familiar and suitably stimulating and safe environment (Cunningham, 2006a; Goodall, 2006).

There is some evidence of increasing interest in the development of dementia special care units (SCU) within hospitals. It is thought that a SCU will offer benefits to people who are cognitively impaired, their families as well as staff and the hospital (Goodall, 2006). The Windows to the Heart Program is an example of the development of an Acute Care Dementia Unit in a 500-bed hospital in New York (Nichols \& Heller, 2002). The program required a purpose built eight-bed unit to be constructed. Staff included a pastoral care worker and an educated multidisciplinary team who promoted a family centred focus in the unit. While outcomes appear to be positive such as fewer restraints, less weight loss, less functional loss and positive responses from those who visit the unit, comparison to a control group, or further research, would give a clearer indication of the program's success.

\section{Internal stressors}

As described earlier, some behaviours displayed by people with dementia may be indicative of unmet needs (Kolanowski et al., 2002; Frazier-Rios \& Zembrzuski, 2005). Unmet needs including physical, social and emotional needs can act as a stressor and lead to anxious and dysfunctional or confused states (McCloskey, 2004). Examples of internal stressors include but are not limited to; pain (Hanley, 2004), dehydration (Archibald, 2006a), inadequate nutrition or hunger (Archibald, 2006b), immobility (Inouye et al., 1999), fatigue (McCloskey, 2004), vision and hearing impairments (Inouye et al., 1999), perceptions of loss (McCloskey, 2004), polypharmacy and other physiological changes and conditions that can contribute to cognitive decline (Hanley, 2004). Interventions can be implemented to reduce the risk of the older person becoming negatively affected by such stressors. Foreman and Zane (1996) recommend the use of preventative strategies while providing symptomatic and supportive care. This approach is well supported by other scholars and is evidenced in the programs developed in hospital settings such as The Hospital Elder Life Program (HELP) (Inouye et al., 2000), which is discussed in a later section.

\section{Family/carer involvement}

Older people who are cognitively impaired and in hospital appear to benefit when a family member or carer participate in aspects of their care. Beneficial family/carer participation includes sharing information with staff. This information may establish the older person's usual behaviour patterns and will help to individualize care plans in order to provide a relatively consistent routine, and help older people with dementia to stay calm and feel secure (McCloskey, 2004). Further support for the importance of family involvement in care is discussed in the following section in a program called creating avenues for relative empowerment (CARE) (Li et al., 2003).

\section{Models of care for older people with dementia in the acute care setting}

Three models developed for use with older people with confusion in acute care, were uncovered: progressively 
W. Moyle et al.

Table 2 Models of care for older people with dementia in the acute care setting

\begin{tabular}{|c|c|c|c|}
\hline Model & Reference & Aim/s of model & Outcome/s \\
\hline $\begin{array}{l}\text { Progressively lowered } \\
\text { stress threshold (PLST) }\end{array}$ & $\begin{array}{l}\text { Hall et al. (1986); } \\
\text { Hall and Buckwalter (1987); } \\
\text { Swanson et al. (1993, 1994); } \\
\text { McCloskey (2004) } \\
\text { Smith et al. (2004) }\end{array}$ & $\begin{array}{l}\text { Advocates a modification of the } \\
\text { hospital environment to take account } \\
\text { of the progressive decline in the } \\
\text { person's cognitive impairment and } \\
\text { to lessen the number of stressors } \\
\text { person is exposed to. }\end{array}$ & $\begin{array}{l}\text { No studies have evaluated PLST } \\
\text { model in acute care. } \\
\text { PLST model in residential care } \\
\text { resulted in increase in social activity, } \\
\text { sleep, dietary intake, and a reduction } \\
\text { in disruptive behaviour. }\end{array}$ \\
\hline $\begin{array}{l}\text { Hospital elder life } \\
\text { program (HELP) }\end{array}$ & $\begin{array}{l}\text { Inouye et al. (2000); } \\
\text { Bradley et al. (2005) }\end{array}$ & $\begin{array}{l}\text { Involves joining of skilled } \\
\text { interdisciplinary team with expertise } \\
\text { in care of older people, to highly } \\
\text { trained and supervised volunteer } \\
\text { staff. } \\
\text { Uses interdisciplinary geriatric } \\
\text { assessment combined with the } \\
\text { delivery of standardized protocols } \\
\text { for the management of six risk } \\
\text { factors for delirium. }\end{array}$ & $\begin{array}{l}\text { HELP resulted in reduction in } \\
\text { delirium, less use of restraints, } \\
\text { greater satisfaction with care and } \\
\text { improved care staff understanding } \\
\text { of care of older people with } \\
\text { confusional states. }\end{array}$ \\
\hline $\begin{array}{l}\text { Creating avenues for relative } \\
\text { empowerment (CARE) }\end{array}$ & Li et al. (2003) & $\begin{array}{l}\text { CARE prepares family caregivers for } \\
\text { common characteristics and } \\
\text { behaviours of hospitalized older } \\
\text { people and provides strategies to } \\
\text { assist them in taking an active role } \\
\text { during the hospitalization. } \\
\text { Intervention includes information } \\
\text { delivered in audio and print formats } \\
\text { and a mutual nurse/family agreement } \\
\text { consisting of family caregiving } \\
\text { activities during hospitalization. }\end{array}$ & $\begin{array}{l}\text { CARE resulted in fewer incidents } \\
\text { of acute confusion and depressive } \\
\text { symptoms and a strengthening of } \\
\text { caregivers' own beliefs about their } \\
\text { understanding of older persons' } \\
\text { behaviour and their ability to care } \\
\text { for them. }\end{array}$ \\
\hline
\end{tabular}

lowered stress threshold (PLST), The HELP and CARE (see Table 2). The first model, the PLST model was first described and reported in 1986 (Hall et al., 1986) in response to caregiver reports that people with dementia exhibited behavioural symptoms, often did not respond to traditional care principles such as reality orientation, and exhibited a progressive lowering of the stress threshold, which created challenges for care provision. The PLST model is underpinned by the theoretical assumption that, although a person's stress threshold is set in adulthood, biological changes created by syndromes such as dementia, can lower the stress threshold and therefore result in dysfunctional behavioural responses. The model advocates a modification of the acute hospital setting, for example, noise reduction and placement of familiar objects to take account of the progressive decline in cognition in the person with dementia and to lessen the number of potential stressors that the person with dementia is exposed to. The model aims to promote more adaptive and functional behaviours by reducing the impact of stress on the person with dementia while maintaining a client-centred approach (Smith et al., 2004).
Six main stressors have been acknowledged as major factors that contribute to the decline of people with dementia from baseline to anxious and more dysfunctional states. These include (i) fatigue; (ii) changes in routine, environment or caregiver; (iii) demands that exceed functional capacity; (iv) multiple and competing stimuli; (v) affective responses to perceptions or loss, including anger; and (vi) physical stressors, such as pain (McCloskey, 2004; Smith et al., 2004). The model sets a framework for the understanding and direction of dementia care that can be applied in a variety of settings. The specific principles developed to deal with the challenges of dementia care in the acute setting are highlighted in Table 3 (McCloskey, 2004; Smith et al., 2004).

While there have been a number of studies exploring the effect of the PLST care principles in a variety of settings (e.g. Hall \& Buckwalter, 1987; Swanson et al., 1993, 1994) its efficacy does not appear to have been tested in acute care. Quasi-experimental research comparing outcomes of the PLST model with traditional models in residential care (Swanson et al., 1993, 1994) suggested the PLST model resulted in an increase in socialization, sleep and dietary 
Table 3 Principles of the progressively lowered stress threshold model

Modify environment to compensate for cognitive losses

Plan and maintain a consistent routine

Modify reality orientation and other therapeutic interventions to incorporate only information needed for safe function

Provide unconditional positive regard
Control factors that increase stress (6 main stressors)

Include regular rest periods to compensate for fatigue and loss of reserve capacity

Remain non-judgemental about behaviours that may seem inappropriate unless there is a threat to safety

Use reassuring forms of therapy, such as music and reminiscence intake and a reduction in disruptive behaviours such as agitation, wandering, and repetitive questioning with a subsequent reduction in psychotrophic medications. A quasi-experimental design was also used in the community to compare an intervention group (PLST) with a control over a 4-year period with 241 caregivers. The PLST intervention was more effective than the comparison approach and promoted greater self-confidence in the caregivers. Given that the model was proposed to assist the acute care environment it is surprising to find that it has not been evaluated in this setting.

Although there are few programs in acute care that specifically focus on care of the person with dementia there are a number of programs that were developed for care of older people whose principles aim to prevent cognitive and functional decline and therefore will benefit older people with dementia. The HELP is a comprehensive model of care for older people who are hospitalized. It aims to maximize independence at discharge; assist with transition from hospital to home and prevent unplanned readmissions (Inouye et al., 2000). The program uses a multi-component intervention strategy, which primarily involves the joining of a skilled interdisciplinary team with expertise in care of older people, to highly trained and supervised volunteer staff and interdisciplinary geriatric assessment combined with the delivery of standardized protocols for the management of risk factors for delirium (Inouye et al., 1999, 2000). Volunteer staff carries out daily orientation, early mobilization, feeding assistance, therapeutic activities, a non-pharmacological sleep protocol, and hearing/vision adaptations.

Inouye et al. (1999) conducted a large prospective controlled clinical trial $(n=852$ ) of people over 70 years of age in a general medicine service. The study used prospective, individual matching to compare people admitted to one intervention (HELP) and two usual care units (control). The authors found that delirium occurred in $9.9 \%$ of the intervention group compared with $15 \%$ of the usual care group (matched odds ratio, 0.60; 95\% confidence interval, 0.39-0.92). Some other benefits included less use of restraints, greater satisfaction with care by both patient and family, improved clinical staff understanding of care of older people and increased communication across the multidisciplinary team.

A further longitudinal qualitative follow up study examined the outcomes and sustainability of the HELP programme in 13 hospitals (Bradley et al., 2005). Bradley et al. (2005) found that three critical factors were necessary in order for the HELP program to be sustained over time. Firstly, the need for ongoing clinical leaders or their replacement if they leave is critical as they play important clinical roles as well as being advocates of the program to senior administration. Secondly, when implementing the program there was a need for some modification and adaptation based on the local constraints and staffing of the hospital. Finally, there is a need for adequate resources and funding that will sustain the program over the long-term. This program again highlights the importance of education for acute care staff in care of older people and the importance of educated and adequately supervised volunteers.

Jones and Borbasi (2006) described the implementation of the HELP program in one South Australian facility. Their descriptive exploratory study of management of people with dementia in acute care described the success of the HELP program in reducing staff burden and a positive cultural change through ongoing staff education and multidisciplinary collaboration. The program resulted in a positive approach to restraint elimination, and proactive patient care management of people with dementia. The benefits of the program seem to be in staff awareness of the special needs of people with dementia and modification of the environment to accommodate these needs.

Creating avenues for relative empowerment was a pilot study carried out in a New York hospital (Li et al., 2003) and was introduced because of the increasing numbers of older patients with both acute and chronic confusion. It aimed to improve outcomes for older people and their family caregivers. A randomized clinical trial was conducted with 49 family caregivers of hospitalized older people. The programme prepared family caregivers for common characteristics and behaviours of hospitalized older people and provided strategies to assist them in taking an active role during the 
hospitalization. The intervention included information delivered in audio and print formats and a mutual nurse/family agreement consisting of family caregiving activities during hospitalization. The control group were given information about hospital services and policies. Participants in the intervention group had fewer incidents of acute confusion and depressive symptoms and there was a strengthening of caregivers' own beliefs about their understanding of older persons' behaviour and their ability to care for them. While the study results were limited by the small sample size use ( $n=49$ ), the authors concluded that improving family caregivers' confidence and knowledge may improve family participation in care of older people and lead to positive outcomes for the older person and caregiver (Li et al., 2003). Other research literature also demonstrates that preparation, support and involvement of family in care of older people with confusion assists in an improvement of patient outcomes and reduces staff and family burden (Hancock et al., 2003).

Although there is acknowledgment for the benefits of effective dementia care in the acute care environment there are a limited number of studies that have tested the efficacy of models of care for chronic confusion in acute care (e.g. Inouye et al., 1999; Li et al., 2003; Bradley et al., 2005). Several of the studies have been limited by the lack of comparison to a control group as well as limited sample sizes, especially where studies have been conducted as a pilot in a small number of units (e.g. Li et al., 2003). In addition the emphasis of the majority of the studies has been on acute confusion rather than dementia. While a large randomized clinical trial demonstrated the effectiveness of the HELP program (Inouye et al., 1999) the uptake of the program seems to have been confined to the USA and Australia. It is concluded that until there is a clear indication of the cost effectiveness of the HELP program the uptake of the program may remain limited. It is apparent that further large-scale research comparing different models of care to a control group is required. In addition given the importance of the family's involvement in the care of older people with confusional states research exploring family engagement is necessary as well as an exploration of the efficacy of the dementia special care units in acute care.

\section{Conclusion}

Within the literature, there are various strategies and models of care designed for older people with dementia in the acute setting. The aim of these models is to improve outcomes for the patient, their family, hospital staff and the hospital. Although all but one of the models would benefit from further research to establish efficacy and effectiveness they all contain similar if not the same principles. The models outlined above all emphaize the importance of staff education, standard clinical protocols and expertise in assisting best practice in care of people with confusional states such as dementia. In addition there is an emphasis on the benefits of supportive environments that meets the physical, emotional and sensory needs of older people. While such principles have been integrated into long term care it appears that the same emphasis has not taken place within acute care settings. However, it was surprising given the increasing numbers of older people with dementia to find a limited evidence base in the literature for care of chronic confusion as a result of dementia.

Acute hospitals constitute a different care environment when compared with residential care homes, a different staff ratio, skills mix and staff education. Whereas staff in care homes are generally aware of the needs of older people acute care staff may not have the education and understanding of older people to ensure their practice has an older person focus. Often people with dementia are 'managed' by an assignment of one staff member to oversee that the person is not disruptive to the ward routine. The perception that the person with dementia is at fault rather than the environment is one of the key principles that Kitwood (1993) sought to challenge. Describing behaviour retrospectively using the antecedents, behaviour and consequences (ABC) analysis of behaviour tool may also help to challenge such assumptions. Such a method may help to stop practitioners' inappropriately labelling and blaming people with dementia, and to explore how they might adapt their practice and the environment rather than to change the person.

The available evidence suggests that there are a number of principles that acute hospitals would do well to incorporate to maximize the care of older people with dementia in acute care. Of prime importance is the development of staff expertise and education in the care of older people with chronic confusion. Further assistance could be made possible through standardized care protocols that include evidence based assessment and detection of confusion tools and that follow a plan of action so that the person is treated and cared for as appropriate. Furthermore, environmental modifications that promote orientation and decrease negative stimulation may assist older people with dementia to wander without feeling confined or being restrained. However, given the relative paucity of studies that test and report on best practice models of care of people with dementia and the dominance of small scale studies, this review of the literature demonstrates the need for further research that aims to identify and evaluate such a model of care. In particular, the authors argue that the review demonstrates the need for 
intervention studies that move beyond the descriptive to an evidence base for care of people with dementia in acute settings. Future research should incorporate the current principles of care of older people with dementia into intervention studies that document the type of intervention used in sufficient detail for others to implement. Although there is an assumption that the principles are common to both acute and chronic care there is limited research to support such a premise and it is timely that such assumptions are tested.

\section{References}

Archibald C. (2006a) Promoting hydration in patients with dementia in healthcare settings. Nursing Standard 20, 49-52.

Archibald C. (2006b) Meeting the nutritional needs of patients with dementia in hospital. Nursing Standard 20, 41-45.

Australian Institute of Health and Welfare (AIHW). (2004) The Impact of Dementia on the Health and Aged Care Systems, AIHW Cat No. Age 37, AIHW, Canberra.

Borbasi S., Jones J., Lockwood C. \& Emden C. (2006) Health professionals' perspectives of providing care to people with dementia in the acute setting: towards better practice. Geriatric Nursing 27, 300-308.

Bradley E., Webster T., Baker D., Schlesinger M. \& Inouye S. (2005) After adoption: sustaining the innovation. A case study of disseminating the Hospital Elder Life Program. Journal of the American Geriatrics Society 53, 1455-1461.

Chrzescijanski D., Moyle W. \& Creedy D. (2007) Reducing dementia related aggression through a staff education intervention. Dementia: The International Journal of Social Research and Practice 6, 271-286.

Courtney M., Rickard C., Vickerstaff J. \& Court A. (2005) Evidencebased nursing practice. Ch. 1. In Evidence for Nursing Practice (Courtney M. ed.). Elsevier, Sydney, pp. 3-21.

Cunningham C. (2006a) Understanding challenging behaviour in patients with dementia. Nursing Standard 20, 42-45.

Cunningham C. (2006b) Meeting the nutritional needs of patients 9 with dementia in hospital. Nursing Standard 20, 45.

Cunningham C. \& Archibald C. (2006) Supporting people with dementia in acute hospital settings. Nursing Standard 20, 51-55.

Dewing J. (2001) Care for older people with a dementia in acute 10 hospital settings. Nursing Older People 13, 18-20.

Draper B. (2004) Dealing With Dementia. A Guide to Alzheimer's Disease and Other Dementias. Allen and Unwin, Sydney.

Eriksson C. \& Saveman B. (2002) Nurses' experiences of abusive/ non-abusive caring for demented patients in acute care settings. Scandinavian Journal of Caring Sciences 16, 79-85.

Feil N. (1972) A new Approach to Group Therapy With the Senile Psychotic Aged. Gerontological Society, Winter Conference, San Juan.

Fick D. \& Foreman M. (2000) Consequences of not recognizing delirium superimposed on dementia in hospitalized elderly individuals. Journal of Gerontological Nursing 26, 30-40.

Fick D. \& Mion L. (2005) Assessing and Managing Delirium in Persons With Dementia. The John A. Hartford Institute for
Geriatric Nursing and the Alzheimer's Association. Vol 1(8). Available at: http://www.hartfordign.org/publications/trythis/fin11 Recog.pdf (accessed 10 January 2007).

Folstein M.F., Folstein S.E. \& McHugh P.R. (1975) Mini-mental state: a practical method for grading the state of patients for the clinician. Journal of Psychiatric Research 12, 189-198.

Foreman M. \& Zane D. (1996) Nursing strategies for acute confusion in elders. American Journal of Nursing. 96, 44-51.

Frazier-Rios D. \& Zembrzuski C. (2005) Communication difficulties: assessment and interventions. Dermatology Nursing 17, 319.

Goodall D. (2006) Environmental changes increase hospital safety for dementia patients. Holistic Nursing Practice 20, 80-84.

Gutterman E., Markowitz J., Lewis B. \& Fillit H. (1999) Cost of Alzheimer's disease and related dementia in managed-medicare. Journal of American Geriatric Society 47, 1065-1071.

Hall G. \& Buckwalter K. (1987) Progressively lowered stress threshold: a conceptual model for care of adults with Alzheimer's disease. Archives of Psychiatric Nursing 1, 399-406.

Hall G., Kirschling M. \& Todd S. (1986) Sheltered freedom: the creation of a special care Alzheimer's unit in an intermediate level facility. Geriatric Nursing 7, 56-63.

Hancock K., Chang E., Chenoweth L., Clarke M, Carroll A. \& Jeon Y.H. (2003) Nursing needs of acutely ill older people. Journal of Advanced Nursing 44, 507-516.

Hanley C. (2004) Delirium in the acute care setting. MEDSURG Nursing 13, 217-225.

Hart B., Birkas J., Lachmann M. \& Saunders L. (2002) Promoting positive outcomes for elderly persons in the hospital: prevention and risk factor modification. AACN Clinical Issues 13, 22-33.

Hunt N. (2007) Setting the backdrop in caring for people with dementia. Nursing Older People 18, 18-19.

Inouye S., Bogardus S., Charpentier P., Leo-Summers L., Campora D., Holford T. \& Cooney L. (1999) A multicomponent intervention to prevent delirium in hospitalized older patients. The New England Journal of Medicine 340, 669-676.

Inouye S., Bogardus S., Baker D., Leo-Summers L. \& Cooney L. (2000) The Hospital Elder Life Program: a model of care to prevent cognitive and functional decline in older hospitalized patients. Journal of the American Geriatrics Society 48, 16971076.

Jimenez-Vilchez A., Roure-Murillo R. \& Vinas-Salas J. (2004) Study of confusion syndrome in patients admitted to a trauma unit: why do elderly patients get disorientation? Journal of Orthopaedic Nursing 8, 4-10.

Jones J. \& Borbasi S. (2006) Dementia related aggression in the acute sector: is a Code Black really the answer? Contemporary Nurse 21, 103-115.

Kitwood T. (1993) Towards a theory of dementia care: the interpersonal process. Ageing and Society 13, 51-67.

Kolanowski A.M., Richards K.C. \& Sullivan S.C. (2002) Derivation of an intervention for need-driven behaviour: activity preferences of persons with dementia. Journal of Gerontological Nursing 28, 12-15.

Lemiengre J., Nelis T., Joosten E., Braes T., Foreman M., Gastmans C. \& Milisen K. (2006) Detection of delirium by bedside nurses using the confusion assessment method. Journal of the American Geriatrics Society 54, 685-689. 
Li H., Melnyk B., McCann R., Chatcheydang J., Koulouglioti C., Nichols 1., Lee M. \& Ghassemi A. (2003) Creating avenues for relative empowerment (CARE): a pilot test of an intervention to improve outcomes of hospitalized elders and family caregivers. Research in Nursing and Health 26, 284-299.

McCarthy M. (2003a) Situated clinical reasoning: distinguishing acute confusion from dementia in hospitalized older adults. Research in Nursing and Health 26, 90-101.

McCarthy M. (2003b) Detecting acute confusion in older adults: comparing clinical reasoning of nurses working in acute, longterm, and community health care environments. Research in Nursing and Health 26, 203-212.

McCloskey R. (2004) Caring for patients with dementia in an acute care environment. Geriatric Nursing 25, 139-144.

Mezey M. \& Maslow K. (2004) Recognition of Dementia in Hospitalized Older Patients. The John A. Hartford Institute for Geriatric Nursing and the Alzheimer's Association. Available at: http:// www.hartfordign.org/publications/trythis/finRecog.pdf (accessed 10 January 2007).

Neal M. \& Barton Wright P. (2003) Validation Therapy for Dementia. Cochrane Database of Systematic Reviews, Issue 3. Art.

12 No: CD001394.

Nichols J. \& Heller K. (2002) Windows to the heart: creating an acute care dementia unit. Journal of Palliative Medicine 5, 181192.

Nolan L. (2006) Caring connections with older persons with dementia in an acute hospital setting - a hermeneutic interpretation of the staff nurse's experience. International Journal of Nursing Older People Nursing 1, 208-215.

Pfaff J. (2002) The geriatric resource nurse model: a culture change. Geriatric Nursing 23, 140-144.
Poole J. (2003) Poole's algorithm: nursing management of disturbed behaviour in older people - the evidence. The Australian Journal of Advanced Nursing 20, 38-43.

Price J.D., Hermans D.G. \& Grimley Evans J. (2005) Subjective Barriers to Prevent Wandering of Cognitively Impaired People. The Cochrane Database of Systematic Reviews 4. Available at: http://gateway.ut.ovid.com/gw1/ovidweb.cgi (accessed 10 January 2006).

Pritchard E. \& Dewing J. (2001) Older people with dementia in acute settings. Nursing Older People 12, 21.

Smith M., Gerdber L., Hall G. \& Buckwalter K. (2004) History, development, and future of the Progressively Lowered Stress Threshold: a conceptual model for dementia care. Journal of the American Geriatrics Society 52, 1755-1760.

Swanson E., Maas M. \& Buckwalter K. (1993) Catastrophic reactions and other behaviours of Alzheimer's residents: special unit compared with traditional units. Archives of Psychiatric Nursing 7 , 292-299.

Swanson E., Maas M. \& Buckwalter K. (1994) Alzheimer's residents cognitive and functional measures: special and traditional care unit comparison. Clinical Nursing Research 3, 27-41.

Tolson D., Smith M. \& Knight P. (1999) An investigation of the components of best nursing practice in the care of acutely ill hospitalized older patients with coincidental dementia: a multi-method design. Journal of Advanced Nursing 30, 1127-1136.

Voyer P., McCusker J., Cole M. \& Khomenko L. (2006) Influence of prior cognitive impairment on the severity of delirium symptoms among older patients. Journal of Neuroscience Nursing 38, 90-101.

Weber J., Coverdale J. \& Kunik M. (2004) Delirium: current trends in prevention and treatment. Internal Medicine Journal 34, 115121. 


\section{Author Query Form}

\section{Journal: $\quad$ OPN}

Article: $\quad 114$

Dear Author,

During the copy-editing of your paper, the following queries arose. Please respond to these by marking up your proofs with the necessary changes/additions. Please write your answers on the query sheet if there is insufficient space on the page proofs. Please write clearly and follow the conventions shown on the attached corrections sheet. If returning the proof by fax do not write too close to the paper's edge. Please remember that illegible mark-ups may delay publication.

Many thanks for your assistance.

\begin{tabular}{|c|c|c|}
\hline $\begin{array}{l}\text { Query } \\
\text { reference }\end{array}$ & Query & Remarks \\
\hline 1 & Au: Please amend/approve the suggested short title. & \\
\hline 2 & $\begin{array}{l}\text { Production Editor: Please supply Submitted for publication, Accepted } \\
\text { for publication dates. }\end{array}$ & \\
\hline 3 & $\begin{array}{l}\text { Au: Chrzescijanski, Moyle \& Creedy, } 2007 \text { has been changed to } \\
\text { Chrzescijanski et al., } 2007 \text { so that this citation matches the list. }\end{array}$ & \\
\hline 4 & $\begin{array}{l}\text { Au: Fick and Forman, }(2000) \text { has been changed to Fick and Foreman } \\
(2000) \text { so that this citation matches the list. }\end{array}$ & \\
\hline 5 & $\begin{array}{l}\text { Au: Peoples, } 1982 \text { not found in the list. Please provide publication } \\
\text { details. }\end{array}$ & \\
\hline 6 & $\begin{array}{l}\text { Au: Robb, } 1986 \text { not found in the list. Please provide publication } \\
\text { details. }\end{array}$ & \\
\hline 7 & $\begin{array}{l}\text { Au: Roseland, } 1997 \text { not found in the list. Please provide publication } \\
\text { details. }\end{array}$ & \\
\hline 8 & $\begin{array}{l}\text { Au: Toseland (1997 not found in the list. Please provide publication } \\
\text { details. }\end{array}$ & \\
\hline 9 & $\begin{array}{l}\text { Au: Cunningham (2006b) not cited. Please cite reference in text or } \\
\text { delete from the list. }\end{array}$ & \\
\hline 10 & $\begin{array}{l}\text { Au: Dewing (2001) not cited. Please cite reference in text or delete } \\
\text { from the list. }\end{array}$ & \\
\hline 11 & $\begin{array}{l}\text { Au: Fick \& Mion (2005) not cited. Please cite reference in text or } \\
\text { delete from the list. }\end{array}$ & \\
\hline 12 & $\begin{array}{l}\text { Au: Please provide publisher address in reference Neal \& Barton } \\
\text { Wright (2003). }\end{array}$ & \\
\hline 13 & $\begin{array}{l}\text { Au: McClosky (2004) has been changed to McCloskey (2004) so that } \\
\text { this citation matches the list. }\end{array}$ & \\
\hline
\end{tabular}




\section{Please correct and return this set}

Please use the proof correction marks shown below for all alterations and corrections. If you wish to return your proof by fax you should ensure that all amendments are written clearly in dark ink and are made well within the page margins.

\begin{tabular}{|c|c|c|}
\hline Instruction to printer & Textual mark & Marginal mark \\
\hline Leave unchanged & ... under matter to remain & ( ) \\
\hline $\begin{array}{l}\text { Insert in text the matter } \\
\text { indicated in the margin }\end{array}$ & $h$ & $\begin{array}{l}\text { New matter followed by } \\
h \text { or } h \otimes\end{array}$ \\
\hline Delete & $\begin{array}{l}\text { I through single character, rule or underline } \\
\text { or }\end{array}$ & $\sigma$ or $\sigma(x)$ \\
\hline $\begin{array}{l}\text { Substitute character or } \\
\text { substitute part of one or } \\
\text { more word(s) }\end{array}$ & I through letter or & $\begin{array}{l}\text { new character / or } \\
\text { new characters / }\end{array}$ \\
\hline Change to italics & — under matter to be changed & $\leftarrow$ \\
\hline Change to capitals & $\equiv$ under matter to be changed & $\equiv$ \\
\hline Change to small capitals & $=$ under matter to be changed & $=$ \\
\hline Change to bold type & $\sim$ under matter to be changed & $\sim$ \\
\hline Change to bold italic & $\bar{\sim}$ under matter to be changed & $\underline{s i n}$ \\
\hline Change to lower case & Encircle matter to be changed & $\Rightarrow$ \\
\hline Change italic to upright type & (As above) & \\
\hline Change bold to non-bold type & (As above) & \\
\hline Insert 'superior' character & $\begin{array}{l}/ \text { through character or } \\
K \text { where required }\end{array}$ & $\begin{array}{l}y^{\prime} \text { or } y \\
\text { under character } \\
\text { e.g. } y^{2} \text { or } y^{2}\end{array}$ \\
\hline Insert 'inferior' character & (As above) & $\begin{array}{l}\lambda \\
\text { over character } \\
\text { e.g. } \hat{\Sigma}\end{array}$ \\
\hline Insert full stop & (As above) & $\odot$ \\
\hline Insert comma & (As above) & , \\
\hline Insert single quotation marks & (As above) & $\begin{array}{l}\dot{y} \text { or } \dot{x} \text { and/or } \\
\dot{y} \text { or } \dot{y}\end{array}$ \\
\hline Insert double quotation marks & (As above) & $\begin{array}{l}\ddot{y} \text { or } \ddot{y} \text { and/or } \\
\ddot{y} \text { or } \ddot{y}\end{array}$ \\
\hline Insert hyphen & (As above) & 1 \\
\hline Start new paragraph & 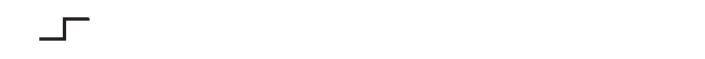 & 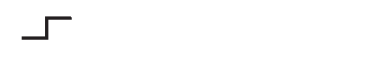 \\
\hline No new paragraph & $\infty$ & $\omega$ \\
\hline Transpose & $\sqcup$ & $\sqcup$ \\
\hline Close up & linking $\bigcirc$ characters & \\
\hline $\begin{array}{l}\text { Insert or substitute space } \\
\text { between characters or words }\end{array}$ & $\begin{array}{l}\text { I through character or } \\
\Lambda \text { where required }\end{array}$ & \\
\hline $\begin{array}{l}\text { Reduce space between } \\
\text { characters or words }\end{array}$ & $\begin{array}{l}\text { between characters or } \\
\text { words affected }\end{array}$ & $\uparrow$ \\
\hline
\end{tabular}

